\title{
Les inégalités sociales de santé des travailleurs immigrés au Québec victimes de lésions professionnelles
}

Social Inequalities in Health of Immigrant Workers in Quebec Victims of Occupational Injuries

Las desigualdades sociales a la salud de los trabajadores emigrantes en Quebec víctimas de lesiones profesionales

Sylvie Gravel, Jacques Rhéaume et Gabrielle Legendre

\section{(2) OpenEdition}

\section{Journals}

Édition électronique

URL : https://journals.openedition.org/remi/5876

DOI : 10.4000/remi.5876

ISSN : $1777-5418$

Éditeur

Université de Poitiers

Édition imprimée

Date de publication : 1 juin 2012

Pagination : $57-80$

ISBN : 979-10-90426-04-7

ISSN : 0765-0752

Référence électronique

Sylvie Gravel, Jacques Rhéaume et Gabrielle Legendre, «Les inégalités sociales de santé des travailleurs immigrés au Québec victimes de lésions professionnelles ", Revue européenne des migrations internationales [En ligne], vol. 28 - n 2 | 2012, mis en ligne le 10 octobre 2012, consulté le 14 avril 2022. URL : http://journals.openedition.org/remi/5876 ; DOI : https://doi.org/10.4000/remi.5876 


\title{
Les inégalités sociales de santé des travailleurs immigrés au Québec victimes de lésions professionnelles
}

\author{
Sylvie GRAVEL ${ }^{1}$, Jacques RHÉAUME ${ }^{2}$ \\ et Gabrielle LEGENDRE ${ }^{3}$
}

\section{INTRODUCTION}

\begin{abstract}
u Québec, depuis 2002, des travaux de recherche ont traité des problèmes de santé et de sécurité des travailleurs immigrés en commençant par l'accès à l'indemnisation et aux services de santé et de réadaptation des victimes de lésions professionnelles $^{4}$ (Gravel, 2006). Les résultats de ces travaux sur l'accès à l'indemnisation ont mené à la réalisation d'autres recherches. Elles ont questionné cette fois les dynamiques de prises en charge des mesures préventives et curatives de santé et sécurité au travail (SST) dans les petites entreprises qui embauchent de façon importante une main-d'œuvre immigrante (Gravel, Rhéaume et Legendre, 2011).
\end{abstract}

Le présent article est la synthèse des résultats de deux études originales, l'une démontrant les problèmes d'accès à l'indemnisation des travailleurs immigrés et l'autre démontrant la faible sensibilisation des travailleurs immigrés à la culture de santé et sécurité au travail au sein des petites entreprises qui les embauchent. Le but de cet article est de démontrer que les travailleurs immigrés présentent un ensemble de caractéristiques

1 Professeure au Département d'organisation et ressources humaines, École des sciences de la gestion, Université du Québec à Montréal ; gravel.s@uqam.ca

2 Professeur Émérite au Département de communication sociale et publique, Université du Québec à Montréal ; rheaume.jacques@uqam.ca

3 MBA Recherche en Administration des affaires, Département d'organisation et ressources humaines, École des sciences de la gestion, Université du Québec à Montréal ; legendregabrielle@yahoo.fr

4 La notion de lésions professionnelles, une terminologie francophone propre au Québec, englobe toutes les pathologies physiques et psychologiques survenues à la suite d'un d'accident ou résultant d'une maladie d'origine professionnelle. Elle recouvre donc la question des accidents du travail et celle des maladies professionnelles. 
qui les placent en situation de précarité à la fois sociale et économique, une situation qui génère des inégalités sociales de santé. Les travailleurs immigrés vivant aujourd'hui au Québec, plus éduqués que ceux des vagues antérieures et soucieux de s'inscrire rapidement et efficacement sur le marché du travail, sont surexposés à des risques professionnels. Ils sont plus fréquemment victimes de lésions et de lésions graves et irréversibles. Ils doivent également surmonter davantage d'obstacles pour bénéficier des indemnités auxquelles ils ont droit.

Pourtant les mesures de protection prévues par les lois de SST, s'adressent à tous les travailleurs sans exception. Bien que le Québec soit doté d'une politique d'accès universel et gratuit aux services de santé et d'une politique de SST, les travailleurs immigrés font face à des inégalités sociales de santé ignorées par ces deux politiques. Au Québec, comme en Amérique du Nord, la santé et la sécurité au travail référent à deux dimensions : l'hygiène et la sécurité. La santé au travail inclut les conditions d'hygiène et de salubrité des lieux de travail qui concernent l'environnement physique de l'entreprise (ventilation, etc.) et les procédés de fabrication (pollution intérieure, etc.). La dimension sécurité fait référence aux risques d'accident ou de maladie inhérents au secteur de production, aux procédés de fabrication et aux produits, comme les risques chimiques, ergonomiques, biologiques, thermiques et ceux liés à l'organisation du travail. Précisons également que chaque province canadienne est dotée d'un système d'indemnisation des travailleurs victimes de lésions professionnelles relativement semblable, un régime complémentaire au système de santé. À la différence des travailleurs européens, notamment en France, les travailleurs canadiens victimes de lésions professionnelles bénéficient de traitements cliniques, de services de réadaptation et de compensations salariales ne relevant pas de la part couverte par l'assurance maladie publique qui offre une couverture de base, mais de celle qui lui est complémentaire. Le système d'indemnisation des travailleurs victimes de lésions professionnelles s'autofinance, comme une mutuelle d'assurance, à partir des cotisations versées obligatoirement par les entreprises et des taux de cotisations calculés selon le risque et les expériences antérieures de réclamations dans l'entreprise et dans l'ensemble du secteur de production.

\section{ÉTAT DES CONNAISSANCES}

Dans la littérature scientifique, un consensus se dessine depuis des décennies indiquant que les travailleurs immigrés sont surexposés aux lésions professionnelles, des lésions généralement plus graves, mais pour lesquelles ils réclament moins souvent (Frumkin, Walker et Pransky, 1999 ; Scherzer, Rugulies et Krause, 2005 ; Brown, Domenzain et Villoria-Siegert, 2002 ; RoSPA, 1992). Au Canada, les travailleurs immigrés n'échappent pas à cette tendance. Hormis les travailleurs hautement qualifiés, la plupart connaissent des conditions d'intégration économique difficiles. Ils acceptent, dans les premières années, des emplois dans des secteurs où les risques d'accidents ou de maladies professionnelles sont parmi les plus élevés (Smith et Mustard, 2010), des emplois qui correspondent rarement à leur niveau de formation (Smith et Mustard, 2010 ; Godin et al., 2004). De plus, ils sont généralement embauchés dans des petites entreprises de moins de cinquante travailleurs, dans lesquelles les conditions de SST sont déficientes, faute de 
structures pour coordonner les actions tant préventives que curatives (Gravel, Rhéaume et Legendre, 2011 ; Berthelette et Planché, 1995).

Ces lésions plus fréquentes sont souvent aussi plus graves, irréversibles, voire fatales, parmi les travailleurs immigrés (Statistics Sweden, 2001 ; Windau, 1997 ; Peek-Asa, Erickson et Krauss, 1999 ; Alcorso, 1988). Par peur de représailles, ils utilisent moins fréquemment les voies de recours (Kosny et al., 2011 ; Scherzer, Rugulies et Krause, 2005 ; Lee, Krause et Goetchius, 2003). Même si les travailleurs immigrés bénéficient d'un accompagnement assuré par des membres de leur communauté pour déposer une réclamation, ces craintes persistent (Lashuay et al., 2002). Ces réticences résultent d'un cumul de problèmes liés aux barrières communicationnelles, à la méconnaissance des droits des travailleurs ainsi qu'aux accompagnements inappropriés à ce public par les services médicaux et juridiques au cours du processus de réclamation (Kosny et al., 2011 ; Henshaw, 2002 ; Dembe et al., 2003). De plus, les délais de procédures de réclamation et les coûts que peut entraîner une contestation en cas de refus ont pour effet que même les travailleurs immigrés les mieux informés s'abstiennent d'engager une demande de réclamation en cas de lésion professionnelle (Herbert, Janeway et Schechter, 1999 ; Teran, Baker et Sum, 2002). Outre leurs craintes, trois types de barrières structurelles font obstacle à l'accès à l'indemnisation des travailleurs démunis dont font partie beaucoup de travailleurs immigrés : 1) celles qui relèvent du milieu de travail (Rosenman et al., 2000) ; 2) celles qui sont liées au système médical (Dembe et al., 2003 ; Rudolph et al., 2002) et aux types de lésions, comme les complications médicales et les rechutes (Himmelstein et Pransky, 2003 ; Pransky et al., 2000) ; 3) et les barrières administratives (Strunin et Boden, 2004). Les quelques études qui se sont intéressées aux obstacles structurels selon l'origine ou le statut des travailleurs ont, entre autres, mis en lumière les difficultés d'accéder aux soins médicaux en raison de la barrière de la langue et des coûts (Kosny et al., 2011 ; Sum, 1996).

Dans le même ordre d'idée, Azaroff, Levenstein et Wegman (2002) ont établi, à partir d'une analyse systématique des dossiers de réclamation traités au cours d'une année par le Bureau of Labor Statistics des États-Unis, que huit filtres interféraient dans le processus d'accès à l'indemnisation, associés à différents acteurs : 1) la banalisation de la gravité de l'événement ou des symptômes de maladie par le superviseur ; 2) les pressions exercées par les collègues ou le syndicat afin d'éviter un temps d'arrêt et de maintenir la cadence de la production ; 3) la crédibilité du diagnostic médical selon l'affiliation du médecin à l'entreprise ou à une clinique indépendante ; 4) les jeux de pouvoir entre le corps médical, les instances décisionnelles et l'employeur pour établir le lien étiologique entre la lésion et le travail ; 5) l'incompréhension par l'employeur et les médecins des enjeux sociaux et financiers de la prise en charge médicale ; 6) la négligence de la surveillance des risques par les services d'inspection; 7) la qualité de la rédaction du premier rapport d'événement (complet et conforme) produit par l'employé et l'employeur ; 8) la résistance du médecin ou sa non collaboration à la procédure de réclamation. Ces filtres sont un bon indicateur des difficultés qui jalonnent les parcours d'indemnisation des travailleurs.

Malgré ces études, les institutions dédiées à la surveillance de la santé des travailleurs des différentes provinces canadiennes et des États américains refusent encore d'analyser les données de lésions professionnelles selon les origines, la langue parlée, le 
statut d'immigration ou tout autre descripteur d'une différentiation ethnique ou même « raciale », et ce, malgré les recommandations d'épidémiologistes réputés (Murray, 2003). Tel que nous l'avons montré dans une étude antérieure, le principal argument est que « tous les travailleurs sont égaux devant la loi, et qu'un tel traitement des données serait discriminatoire » (Gravel, Boucheron et Kane, 2003 : 7). De plus, la surveillance de la SST dans les petites entreprises (PE) qui embauchent des immigrés est une problématique non documentée, sauf dans les entreprises saisonnières qui font appel à des travailleurs étrangers temporaires (Underhill et Quinlan, 2011), bien que l'embauche des travailleurs immigrés soit une solution utilisée pour répondre à la pénurie de main-d'œuvre dans toutes les PE, même non saisonnières. Ce phénomène est présent dans plusieurs des pays de l'OCDE (Vincent et Gaudreault, 2011). Soulignons que la majorité des entreprises, même dans les pays de l'OCDE, comptent moins de cinquante employés (Eakin, Lamm et Limborg, 2000). Au Canada, 95 \% des entreprises sont de petite taille (Statistique Canada, 2010). Dans la seule région de Montréal, on compte 7698 petites entreprises, dont $29 \%$ emploient une main-d'œuvre n'ayant pas une connaissance fonctionnelle des deux langues officielles et 20,3\% sont administrées par des propriétaires s'exprimant dans une langue autre que le français et l'anglais (Bouchard, 2008). En dépit de leur présence imposante dans les petites entreprises, nous connaissons peu de choses sur la participation des travailleurs immigrés aux mesures de SST dans leur environnement de travail et sur la compréhension qu'ils ont de leurs droits et de leurs obligations. Certains travaux ont souligné des lacunes importantes dans les activités préventives de santé et sécurité au travail (Henshaw, 2002) et dans l'accès à l'indemnisation des travailleurs immigrés (Kosny et al., 2011 ; Gravel, 2006 ; Brown, Domenzain et Villoria-Siegert, 2002). La dynamique de prise en charge de ces questions dans un contexte multiethnique constitue rarement un objet d'étude.

L'absence de participation des travailleurs immigrés à la culture de la SST s'explique en partie par les contraintes de gestion d'une petite entreprise (Gravel, Rhéaume et Legendre, 2011). Aux prises avec des fluctuations du marché économique menaçant leur survie, les PE consacrent peu de temps et de moyens aux pratiques préventives de santé et de sécurité au travail, conjuguant ainsi plusieurs facteurs de risque (Eakin, Lamm et Limborg, 2000 ; Lansdown, Deighan et Brotherton, 2007). Moins assujetties aux mesures de contrôle et à la règlementation, les $\mathrm{PE}$ bénéficient de moins d'encadrement par des institutions externes, comme les associations sectorielles ou patronales (Quinlan et Mayhew, 2000 ; Lansdown, Deighan et Brotherton, 2007 ; Mendeloff et al., 2006). Les sanctions ou les amendes imposées seraient les principales motivations des patrons des petites entreprises à se conformer à la réglementation (Amodu, 2008). Compte tenu de leurs ressources internes limitées, ces entreprises utiliseraient les services externes gratuits, dont les services en ligne qui ont l'avantage d'être anonymes (Lansdown, Deighan et Brotherton, 2007 ; Desmarais, 2004 ; Health Safety Executive, 2005).

Maints travaux ont démontré les mauvaises conditions de SST des travailleurs immigrés (Kosny et al., 2011 ; Smith et Mustard, 2009 ; Smith et Mustard, 2010 ; Smith, Kosny et Mustard, 2009 ; Brown, Domenzain et Villoria-Siegert, 2002). Néanmoins, ce n'est qu'à la suite d'un tragique accident impliquant des travailleurs immigrés qu'un groupe d'experts canadiens en santé et sécurité au travail a inventorié les activités préventives s'adressant spécifiquement à ces travailleurs (Kosny et al., 2011). Leurs recommandations visent l'adaptation du matériel d'information et de formation aux travailleurs 
immigrés récents et allophones, et l'inclusion, dans les formulaires de demande d'immigration, d'informations sur les droits et obligations en SST à l'intention des travailleurs (Kosny et al., 2011). Une série d'actions préventives s'adressant à ces travailleurs ont été recensées, mais toutes ciblent les travailleurs en recherche d'emploi ou en processus d'immigration. Peu visent les travailleurs immigrés d'ores et déjà en emploi (Kosny et al., 2011 ; WCB update, 2008). Or, les immigrés, soucieux de s'insérer sur le marché du travail, sont peu attentifs à ces avis et consignes, sauf lorsqu'ils y sont confrontés en cours d'emploi. Souvent anxieux de conserver leur emploi, ils sont peu enclins à signifier à leur superviseur les risques, et encore moins à exercer leur droit de refus d'exécuter une tâche dangereuse. L'urgence à s'insérer économiquement les conduit à occuper des emplois aux conditions précaires et risquées. Ils y sont surexposés aux lésions professionnelles, se blessent plus souvent et peinent à être indemnisés. De plus, ils travaillent dans des petites entreprises dont le souci se limite à l'observance des normes minimales prescrites par les lois de SST. Bien que tous ces travaux indiquent des pratiques qui pénalisent les travailleurs vulnérables, parmi lesquels les immigrés sont nombreux, les résultats sont rarement analysés comme des inégalités sociales de santé. En fait, l'analyse des inégalités sociales se focalise généralement sur deux dimensions : l'une matérielle (revenu, logement) et l'autre sociale (éducation, accès aux services de santé, réseau social) (Pampalon et Raymond, 2003 ; Pampalon et al., 2008). Certes, les conditions de travail, dont la rémunération, sont prises comme une dimension des disparités matérielles, mais les conditions de SST sont rarement abordées comme une autre manifestation des inégalités (Berthelette et Planché, 1995). Pourtant, les travaux sur la santé et la sécurité des travailleurs immigrés soulèvent des problèmes d'inégalités à la fois matérielles et sociales. Les difficultés d'accès à l'indemnisation, la sous-déclaration, les craintes de pertes de revenus ou même du lien d'emploi sont des indicateurs indirects de la précarité chez les travailleurs immigrés victimes de lésions professionnelles, une dimension certes plus complexe que le revenu. Dans le même ordre d'idées, la méconnaissance qu'ont les immigrés des droits et des obligations en matière de SST pour exercer correctement leurs possibilités de recours aux services d'indemnisation et de réadaptation, est également une forme complexe d'inégalité sociale (Gravel et al., 2010). La pénalisation sociale des travailleurs immigrés victimes de lésions professionnelles face au système d'indemnisation est d'autant plus criante qu'ils sont généralement surqualifiés pour les emplois qu'ils occupent, des emplois d'insertion économique, des emplois de survie, souvent des emplois de manœuvre, pour lesquels ils n'ont ni les savoirs faire, ni l'expérience (Kosny et al., 2011). Ceci reflète un aspect des politiques migratoires canadiennes qui, au cours des années 1990, sélectionnaient les candidats à l'immigration en s'appuyant sur un système de points favorable aux candidats ayant fait des études universitaires, sans ajustement aux besoins du marché du travail ${ }^{5}$.

Ce constat remet en question les politiques de sélection et d'insertion des immigrés ainsi que les politiques économiques qui s'appuient sur l'immigration pour combler les besoins de main-d'œuvre et assurer la croissance des sociétés des pays de l'OCDE. Il souligne la responsabilité des politiques sociales de protection des travailleurs qui ignorent les disparités entre les travailleurs, disparités liées entre autres aux conditions d'insertion des migrants sur le marché du travail. Ce débat d'équité s'inscrit dans la

5 Notons cependant que, depuis le milieu des années 2000, ce critère favorisant les détenteurs de titres universitaires est en révision. 
théorie des « capability» (capabilités) d'Armataya Sen (2009) où la lutte contre les inégalités sociales ne vise pas l'instauration d'un revenu minimal de subsistance, mais bien l'égalité des opportunités pour combler les aspirations sociales et matérielles motivées par les capacités de chacun.

Souhaitant contribuer au débat sur les inégalités sociales de santé touchant les travailleurs immigrés, notre équipe de recherche a tenté de documenter les deux questions de recherche suivantes:

- Quelle est l'ampleur des problèmes d'accès à l'indemnisation des travailleurs immigrés comparativement aux travailleurs non immigrés?

- Quelles sont les stratégies favorables à l'implantation de mesures préventives en matière de santé et de sécurité au travail dans les petites entreprises qui embauchent des travailleurs immigrés?

\section{MÉTHODOLOGIE}

Les réponses à ces deux questions puisent leurs sources dans deux recherches menées successivement, entre 2002 et 2011, avec des méthodologies distinctes.

\section{Étude portant sur l'accès à l'indemnisation}

La première étude, menée de 2002 à 2004, portait sur les facteurs facilitant et limitant l'accès aux services d'indemnisation et sur les interactions entre les acteurs aux diverses étapes du processus d'indemnisation (Patry et al., 2005). Deux vagues d'entrevues individuelles ont été réalisées auprès d'un échantillon non probabiliste de 104 travailleurs montréalais, soit cinquante-trois travailleurs immigrés et cinquante et un non immigrés. La première série d'entrevues, centrées sur les démarches d'indemnisation, a duré en moyenne $1 \mathrm{~h} 45$, et la seconde, dédiée aux conditions de retour au travail, quarante-cinq minutes. Tous les sujets avaient fait une demande de consultation médicale ou juridique. Les travailleurs étaient sollicités par l'intermédiaire des services cliniques et juridiques qu'ils avaient consultés. Les critères d'inclusion à l'échantillon étaient d'avoir fait une demande de réclamation pour une lésion professionnelle survenue au cours des deux années précédant l'entrevue, d'être embauché par une entreprise au moment de la réclamation, d'être âgé de dix-huit à soixante-cinq ans et d'être au Canada depuis moins de vingt ans pour les sujets immigrés. Cette définition inclut les travailleurs de toute origine ethnique et « raciale », les allophones ou les travailleurs maîtrisant peu les langues officielles. Elle exclut les travailleurs ayant immigré de longue date, considérant qu'ils sont aussi familiers avec les structures sociales que tous les travailleurs, par exemple avec le système d'indemnisation. Les entrevues ont été menées dans la langue maternelle du travailleur par une équipe d'intervieweurs multilingues ou accompagnés d'un interprète lorsque l'équipe ne pouvait assurer la traduction. Les données ont été traitées par les logiciels SPAD et SPSS, avec le test statistique $t$ de Student pour tester l'égalité des deux moyennes entre migrants et non-migrants. 


\section{Étude portant sur les dynamiques de prise en charge de la SST dans les petites entreprises du secteur industriel qui embauchent des travailleurs immigrés}

La deuxième étude, menée de 2008 à 2011, constitue une recherche prospective sur les dynamiques de prise en charge de la SST dans les petites entreprises et elle comporte deux volets (Gravel, Rhéaume et Cyr, 2008) : 1) un volet entreprise portant sur $28 \mathrm{PE}$; 2) un volet travailleur reposant sur un questionnaire auto-administré auprès de 171 travailleurs employés dans les petites entreprises échantillonnées. Les stratégies de recrutement des participants pour ces deux volets ont été différentes.

Les vingt-huit entreprises relèvent du secteur privé, sans égard à la présence syndicale, embauchant entre dix et cinquante travailleurs. L'échantillon comprend deux groupes : les PE d'observation $(n=19)$ et les PE de comparaison $(n=9)$. Les PE d'observation ont une main-d'œuvre composée d'au moins un quart de travailleurs immigrés, nés hors du Canada. À l'inverse, les PE du groupe de comparaison ont une main-d'œuvre composée d'au moins trois quarts de travailleurs nés au Canada. Les critères d'inclusion des PE étaient : 1) d'être situées dans la région métropolitaine de Montréal et 2) d'avoir bénéficié d'une intervention de l'équipe de professionnels de SST des services publics au cours de l'année. La grille d'entrevue, préalablement transmise aux responsables de la santé et la sécurité au Travail des petites entreprises, les guidait sur les informations recherchées. La plupart ont utilisé un registre interne des employés pour identifier, pour nos besoins, le nombre de travailleurs nés à l'étranger, incluant ceux ayant grandi au Canada, puisqu'il ne s'agit pas d'une information validée par des documents officiels. Les entreprises étaient sollicitées par l'entremise des Centres locaux des services sociaux et de santé qui offrent gratuitement des services-conseils en SST.

Parmi les vingt-huit PE participant au premier volet, dix-neuf ont accepté de transmettre le questionnaire auto-administré à leurs salariés. La sélection des travailleurs immigrés a été indépendante du statut sur le territoire (citoyen, immigrant reçu, réfugié ou en attente de statut) et de la durée de séjour au Canada, en considérant ces données comme non pertinentes et potentiellement discriminatoires. Au total, 171 travailleurs ont rempli le questionnaire à choix de réponses multiples, complété en quelques minutes (douze à quinze minutes). Ce questionnaire était disponible en quatre langues : français, anglais, espagnol et arabe. Les questionnaires dans les diverses versions étaient acheminés par courrier aux entreprises, selon le nombre prédéterminé par le responsable de la santé et de la sécurité au travail de chaque établissement. Le questionnaire, qui ne permettait pas d'associer le travailleur-répondant à son entreprise, était accompagné d'une enveloppe de retour pré-adressée à l'équipe de recherche.

\section{RÉSULTATS}

Cet article présente donc les résultats de travaux de recherche portant sur deux questions complémentaires relatives à la santé des travailleurs immigrés : l'indemnisation des travailleurs victimes de lésions professionnelles et les dynamiques de prévention dans les usines qui les embauchent. 


\section{Accès à l'indemnisation des travailleurs immigrés victimes de lésions professionnelles}

À la première question : «Quelle est l'ampleur des problèmes d'accès à l'indemnisation des travailleurs immigrés comparativement aux travailleurs non immigrés ?", une série de données indiquent des écarts significatifs d'accès à l'indemnisation entre les travailleurs immigrés et non immigrés, des écarts qui sont préoccupants.

\section{Analyse des difficultés des parcours d'indemnisation selon des experts en SST}

Les premiers résultats, traités par SPSS, sont des évaluations de difficultés d'accès à l'indemnisation, établies à partir de scores attribués par des experts au parcours de chacun des travailleurs. Il s'agit d'un score moyen pondéré attribué par trois groupes d'experts en SST : des juristes, des médecins et des gestionnaires de programme d'indemnisation et de réadaptation ${ }^{6}$. Les résultats indiquent que les parcours d'indemnisation des travailleurs immigrés de l'échantillon sont jugés plus difficiles (Graphiques 1 à 3 ) sous tous les angles d'évaluation. Dans les trois graphiques, les scores de difficultés sont en abscisse et le nombre des travailleurs en ordonnée. Sous l'angle médical (Graphique 1) et administratif (Graphique 3), la distribution des scores suit une courbe normale avec un décalage entre les courbes des travailleurs immigrés et non immigrés, indiquant des niveaux de difficulté plus grands chez les travailleurs immigrés. Cependant, les différences ne sont significatives que pour l'évaluation réalisée sous l'angle administratif $(t=-2,4)$, contrairement à celle qui est réalisée sous l'angle médical $(\mathrm{t}=-0,97)$. La distribution des scores de difficultés sous l'angle juridique (Graphique 2) est asymétrique et significative $(\mathrm{t}=-2,8)$. Au total, si les travailleurs non immigrés éprouvent des difficultés importantes d'accès au système d'indemnisation, les travailleurs immigrés rencontrent toujours de plus grandes difficultés que les premiers.

6 Le groupe d'experts médicaux en santé et sécurité au travail comprenait un médecin ergonome, un conseiller en indemnisation et un anthropologue médical. Il n'y avait qu'un expert en droit, un juriste en droit de la santé et la sécurité au travail. Et le groupe d'experts des aspects administratifs était composé d'un conseiller aux services à la clientèle, un chercheur spécialiste des programmes de maintien du lien d'emploi pour les travailleurs victimes de lésions professionnelles, un chercheur spécialiste des questions d'inégalités sociales et de santé chez les prestataires d'aide sociale et d'assurance-emploi, et un médecin-conseil attaché à l'instance juridique qui reçoit les demandes de mise en appel des décisions administratives en santé et sécurité au travail. Les scores de difficultés pour chacun des sujets de l'échantillon ont été calculés à partir des critères retenus par les groupes d'experts. Les scores attribués par les experts médicaux se basaient sur trente-trois critères correspondant à la présence ou l'absence de difficultés. Par exemple, pour « la réclamation initiale était-elle conforme à l'événement», oui (score 0 ), non (score 1). Le score maximal associé aux experts médicaux était de 33. L'évaluation faite sous l'angle juridique a été effectuée par un juriste. Celui-ci a relu toutes les entrevues de l'enquête et a attribué un score global à chaque sujet en tenant compte de trente-quatre critères, mais pas nécessairement les mêmes que les experts médicaux. Le score maximum attribué par l'expert du droit était de 10. L'évaluation faite sous l'angle administratif considérait treize critères en leur attribuant une valeur variant d'un à quatre pour chaque critère, avec un score maximal théorique de 65. Ensuite, ces trois scores d'experts médicaux, juridiques et administratifs ont été pondérés. 
Graphique 1 : Évaluation selon l'angle médical : comparaison des scores de difficultés des parcours d'indemnisation selon le statut d'immigration

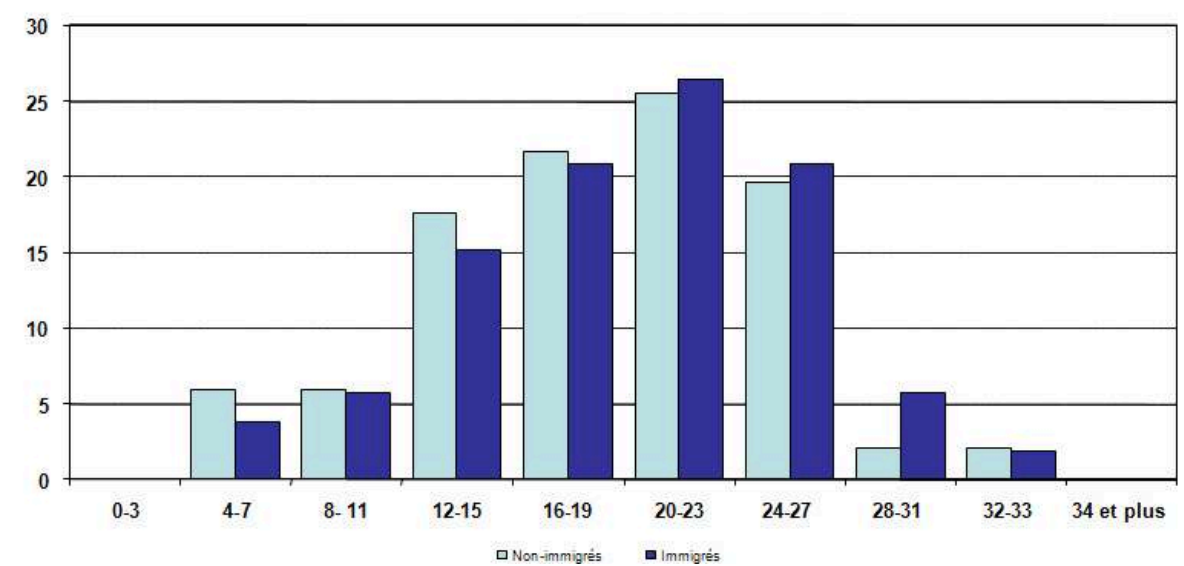

Score moyen (écart-type) immigrés : $19,8(6,4)$; non-immigrés : 18,6 $(6,2)$ $\mathrm{t}=-0,97$, non significatif

Score maximal sur 50

Graphique 2 : Évaluation selon l'angle juridique : comparaison des scores de difficultés des parcours d'indemnisation selon le statut d'immigration

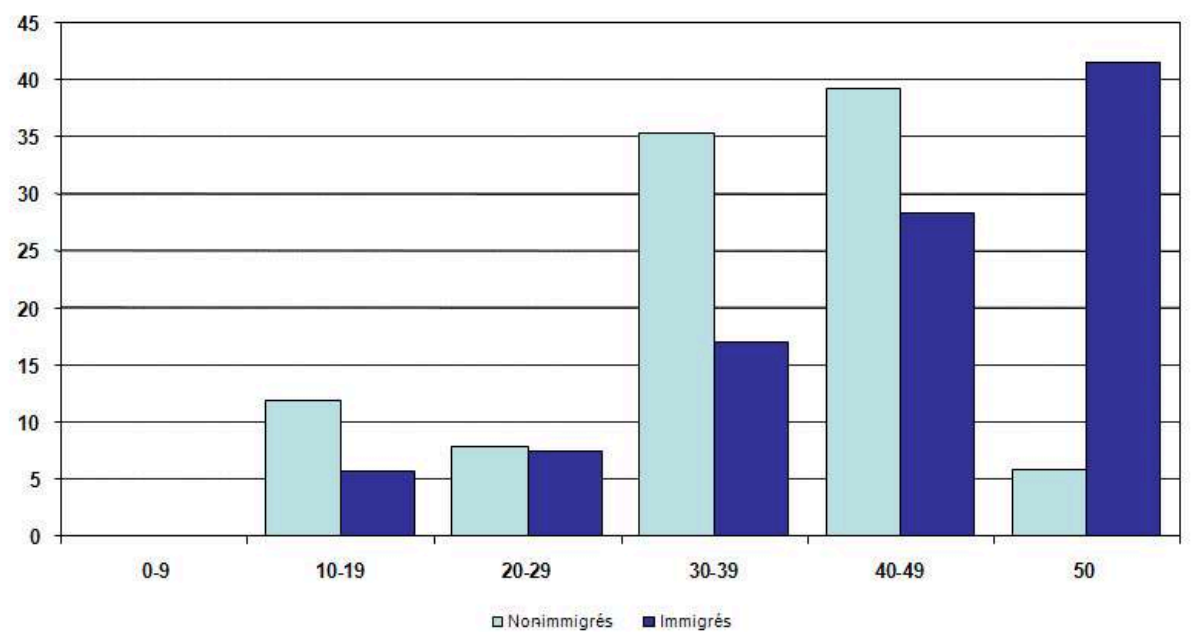

Score moyen (écart-type) immigrés : 40,7 $(10,8)$; non-immigrés : $34,5(10,7)$ $\mathrm{t}=-2,8$, significatif

Score maximal sur 50 


\section{Graphique 3 : Évaluation selon l'angle administratif : comparaison des scores de difficultés des parcours d'indemnisation selon le statut d'immigration}

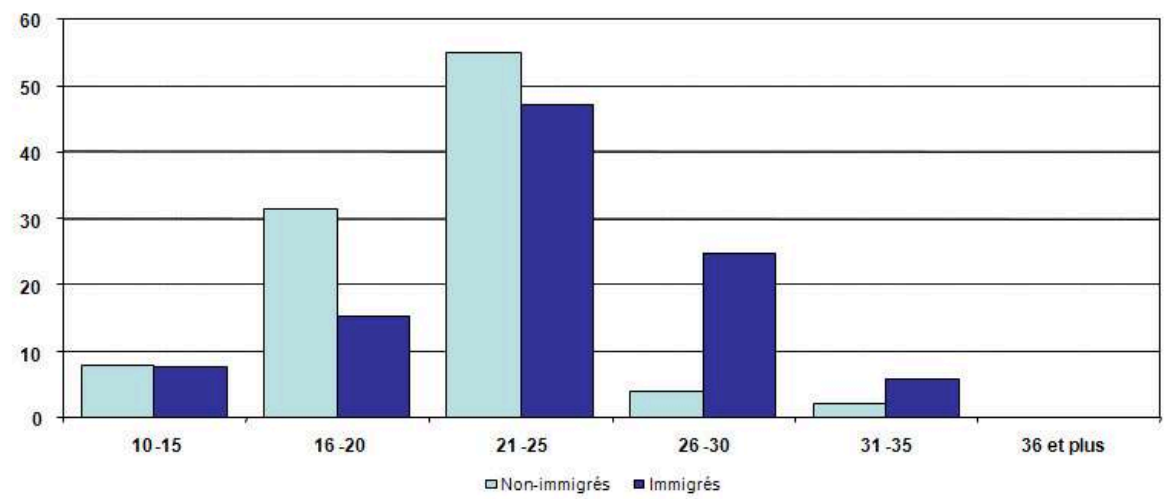

Score moyen (écart-type) immigrés : $23,2(4,8)$; non-immigrés : $20,9(3,4)$

$\mathrm{t}=-2,4$, significatif

Score maximal sur 50

Les tests d'égalité des moyennes des variables associées aux scores de difficultés d'accès à l'indemnisation indiquent que le type de lésion (maladie ou accident), la scolarité ( $<12$ ans d'études et $\geq 12$ ans) et le statut (immigré, non immigré) sont associés de façon significative aux difficultés. Les travailleurs qui ont une faible scolarité $(<12$ ans) ou qui ne s'expriment pas dans l'une ou l'autre des langues officielles ont des scores statistiquement plus élevés dans les parcours difficiles selon les évaluations réalisées sous les angles médical $(p=0,02)$ et administratif $(p=0,02)$. Les médecins et les agents d'indemnisation, qui sont les premières personnes dans le parcours à qui les travailleurs doivent décrire les circonstances entourant l'accident ou l'apparition des symptômes, sont à même de constater les limites des travailleurs peu scolarisés à relater les événements et à communiquer avec les instances décisionnelles. Les travailleurs souffrant de maladies professionnelles ont des scores significativement plus élevés que ceux qui sont victimes d'un accident ( $\mathrm{p}=0,03)$, selon l'évaluation faite sous l'angle juridique. Les travailleurs souffrant d'une maladie professionnelle doivent démontrer que leur état de santé n'est pas lié à une condition personnelle antérieure, mais aux risques particuliers du travail. La démonstration étant souvent difficile à faire, ils ont probablement recours à des conseillers juridiques pour étayer les preuves. Les travailleurs immigrés de l'échantillon ont des scores aux difficultés d'indemnisation qui sont statistiquement plus élevés selon les évaluations faites sous les angles juridique $(p=0,004)$ et administratif $(p=0,007)$ et dans la synthèse des opérations $(\mathrm{p}=0,009)$.

Pour les travailleurs qui ont participé à cette étude, le statut d'immigration est associé aux difficultés d'accès à l'indemnisation lorsque cette évaluation est faite sous les angles juridique et administratif, alors que la faible scolarité est prépondérante sous les angles médical et administratif, et le type de lésion, sous l'angle juridique. Autrement dit, un juriste du droit du travail et un gestionnaire reconnaissent davantage la précarité du 
statut d'immigration comme une barrière d'accès à l'indemnisation, alors que le médecin sera davantage préoccupé par le peu d'éducation du travailleur pour accéder adéquatement au système d'indemnisation. Idéalement, le travailleur devrait maîtriser toutes les étapes $\mathrm{du}$ processus d'indemnisation et les règles administratives pour se voir reconnaître ses droits et maintenir son lien avec son employeur comme employé régulier ou contractuel. Or, l'incompréhension des procédures et des délais est souvent à l'origine du refus dès la première instance décisionnelle d'indemnisation, refus qui aura une incidence sur toutes les autres étapes du processus d'indemnisation.

\section{Analyse de correspondances des obstacles à l'indemnisation}

L'analyse de correspondances est une méthode exploratoire qui sert à positionner un sujet dans un espace tridimensionnel où se superpose un ensemble de caractéristiques le qualifiant (Benzécri, 1994). Le traitement des données par le logiciel SPAD a permis de calculer la contribution des variables actives et illustratives (équivalentes aux variables dépendantes et indépendantes) et de retenir celles qui sont porteuses de sens dans l'interprétation des correspondances. Les variables actives utilisées regroupent quatre dimensions du parcours de l'indemnisation : a) parcours médical (quatre variables), par exemple : diagnostic reçu (précis ou imprécis), complications médicales ou psychologiques (oui, non); b) parcours administratif (onze variables), par exemple : aide obtenue pour compléter la réclamation (oui, non), personnes ayant complété la réclamation (travailleur, employeur, tierce personne) ; c) parcours juridique (sept variables), par exemple : présence de témoins lors des événements (oui, non), contestation de l'employeur (oui, non) ; d) retour au travail (six variables) par exemple : retour au travail (oui au même poste, oui mais congédié par la suite, non invalide), retour progressif (oui, non). Les sept variables illustratives retenues sont : le statut d'immigration (immigré, non immigré), la durée de séjour au Canada (dix ans et moins, onze ans et plus), les années de scolarité ( $<12$ ans, $\geq 12$ ans), la langue maternelle (français ou anglais, espagnol, autre), les difficultés à se faire comprendre (oui, non), les difficultés à comprendre la correspondance écrite (oui, non) et les incompréhensions lors des procédures (oui/non) (Gravel, 2006).

L'analyse de correspondances du parcours médical indique que les travailleurs immigrés vont connaître davantage de complications médicales et psychologiques à la suite d'une première lésion, complications découlant d'un diagnostic initial imprécis. Les diagnostics imprécis sont ici associés aux travailleurs immigrés ayant de la difficulté à se faire comprendre lors des investigations médicales.

L'analyse de correspondances du parcours juridique démontre que les pressions exercées par l'employeur sont plus fréquentes lorsqu'il s'agit des travailleurs immigrés, et elles se traduisent par des taux de refus plus élevés. Même si les travailleurs immigrés vont chercher l'aide d'un conseiller juridique, ce n'est pas nécessairement pour faire appel de la décision, mais plutôt pour en comprendre les termes. Par crainte de perdre leur emploi, les travailleurs immigrés tendent à se conformer à la décision initiale du tribunal.

L'analyse de correspondances du parcours administratif révèle que les travailleurs immigrés ont du mal à comprendre les procédures administratives de l'indemnisation. Dès les premières étapes du processus, les travailleurs dont la langue maternelle est 
autre que le français ou l'anglais, sont désavantagés. Ils déclarent rarement leur lésion le jour même ; ils le font généralement dans les semaines suivantes, souvent au-delà des six mois réglementaires lorsqu'ils souffrent d'une maladie professionnelle. De plus, ils ont souvent recours à une tierce personne - un membre de leur famille, un voisin ou un membre de leur communauté - pour rédiger leur réclamation. Ces personnes ne sont pas nécessairement mieux informées des procédures. Les termes juridiques expliquant la décision sont rarement clairs pour les travailleurs immigrés. Mentionnons que l'instance administrative de protection des travailleurs qui détermine si un travailleur est admissible aux indemnités ou aux services de réadaptation échange avec les travailleurs essentiellement par courrier ou par Internet. Or, la très grande majorité des travailleurs de l'échantillon, immigrés ou non, scolarisés ou non, ne comprend pas cette correspondance rédigée uniquement en des termes juridiques.

L'analyse de corrélations du retour au travail indique que les travailleurs immigrés n'ont bénéficié ni d'un retour progressif ni du soutien de leur employeur. Bien qu'ils soient retournés au travail, les travailleurs immigrés ont tous été par la suite licenciés ou congédiés, contrairement aux travailleurs non immigrés. Ils n'ont pas bénéficié de conditions favorables à la réintégration comme le prescrit la loi sur le retour progressif et l'allégement de la tâche. Un travailleur souffrant d'une maladie professionnelle a plus de difficultés à conserver son emploi à la suite d'une indemnisation, ce qui est doublement pénalisant pour les travailleurs immigrés qui tardent à déclarer ce type de lésions et qui misent sur les congés pour se rétablir. Les résultats de l'analyse de correspondances indiquent qu'il y a des associations importantes entre le statut de travailleur immigré et les difficultés de parcours d'indemnisation, et ce, aux diverses étapes du processus.

\section{Analyse de régression logistique des facteurs prédictifs du refus d'indem- nisation et de retour au travail chez les travailleurs immigrés}

Afin de contrôler certaines variables confondantes pouvant interférer sur les associations significatives, une analyse de régression sur le logiciel SPSS a été réalisée à partir de deux variables dépendantes - l'indemnisation (acceptée, refusée) et le retour au travail (oui, non) - et de huit variables indépendantes : les difficultés financières (oui, non) ; le nombre de personnes à leur charge ; la durée de la scolarité ( $<12$ et $\geq 12$ ans) ; les difficultés à comprendre la correspondance écrite (oui, non) ; le type de lésion (accident, maladie) ; le nombre d'employés dans l'entreprise $(<50,50 \text { et plus })^{7}$; et le statut migratoire (immigré, non immigré). Ce modèle de régression a été construit à partir des résultats de l'analyse de correspondances.

7 Selon la Loi sur la santé et la sécurité au travail, l'obligation d'un employeur à reprendre un employé victime de lésions professionnelles varie selon la taille de son entreprise. Dans une petite entreprise, un travailleur victime de lésions professionnelles dispose d'une année pour se prévaloir de son droit à la réintégration, alors que ceux qui sont embauchés dans une moyenne ou une grande entreprise ont deux ans pour se prévaloir de ce droit (Lois et règlements du Québec et du Canada, 2003; article 240). Cette règlementation varie d'un pays à l'autre selon les limites de la taille des entreprises. Par exemple, en France, la très petite entreprise compte moins de vingt personnes, alors qu'au Québec le plafond est fixé à moins de onze employés.

REMI 2012 (28) 2 pp. 57-80 
Les résultats démontrent plusieurs écarts entre les travailleurs dont certaines caractéristiques sont associées aux immigrés, selon le niveau de signification statistique que nous avons fixé à $\leq p=0,05$. Les travailleurs ayant des difficultés financières ont de trois à quatre fois plus de probabilité de voir leur demande d'indemnisation refusée que les travailleurs n'ayant pas de problème financier $(\mathrm{p}=0,02)$. Les résultats indiquent également que le type de lésion $(\mathrm{p}=0,08)$ a tendance à affecter l'accès à une indemnité : le travailleur victime d'un accident a trois fois moins de risque de se faire refuser que celui qui est victime d'une maladie professionnelle. Le statut migratoire $(p=0,1)$ influe sur le retour au travail : les travailleurs non immigrés ont deux fois plus de chance de retourner au travail que les travailleurs immigrés. Les travailleurs victimes d'un accident professionnel $(p=0,16)$ auraient également deux fois plus de chance de retourner au travail que ceux qui sont victimes de maladies professionnelles.

Les analyses sur l'accès à l'indemnisation des travailleurs immigrés ont permis d'établir que les difficultés sont cumulées tout au long du parcours. Le résultat le plus préoccupant reste l'ampleur du nombre de licenciements des travailleurs immigrés à la suite d'une lésion professionnelle. Les immigrés de l'étude sont embauchés, comme dans plusieurs études américaines, dans des secteurs industriels où la prévalence des lésions est fréquente et, lorsqu'ils réintègrent leur travail à la suite d'une lésion, ils sont plus susceptibles de perdre leur emploi parce qu'ils sont jugés moins productifs. Bien que la prévalence des lésions professionnelles graves et irréversibles chez les travailleurs immigrés soit supérieure, peu d'entre eux ont recours aux indemnités par crainte ou par ignorance. Et lorsqu'ils s'en prévalent, ils le font généralement dans des conditions difficiles, en connaissant mal ou pas du tout les règles et les procédures. Les critères pour juger de leurs difficultés d'accès à l'indemnisation sont complexes et font référence à des dimensions et à des acteurs issus du système de santé et du marché du travail. Le licenciement des travailleurs immigrés à la suite d'une lésion professionnelle engendre un appauvrissement qui génère d'autres inégalités sociales de santé. Sachant que ces travailleurs sont embauchés quasi exclusivement dans les petites entreprises où les mesures de SST sont rares ou inappropriées, nous étions soucieux de connaître les dynamiques de prévention en matière de SST dans ces entreprises. Nous avons réalisé une seconde étude pour documenter ces dynamiques.

\section{Dynamiques de prévention dans les entreprises qui embauchent les travail- leurs immigrés}

Pour explorer notre deuxième question : «Quelles sont les stratégies favorables à l'implantation de mesures préventives de la SST dans les petites entreprises qui embauchent des travailleurs immigrés ? », deux traitements de données ont été réalisés.

Une première analyse compare les pratiques de santé et sécurité au travail, à partir d'entretiens menés auprès des responsables de la santé et de la sécurité au travail, dans les PE embauchant majoritairement des immigrés $(n=19)$ et dans celles qui en embauchent peu ou pas $(n=9)$. Un peu plus de la moitié (dix sur dix-neuf) des entreprises du premier groupe sont homogènes sur le plan ethnique, c'est-à-dire que les dirigeants, les superviseurs et les travailleurs sont issus des mêmes pays d'origine : Inde, Congo, Liban, 
Roumanie, etc. On compte également environ un quart de petites entreprises dont les dirigeants et les superviseurs d'origine canadienne emploient des travailleurs de diverses origines (Haïtien, Coréen, Marocain et Iranien). Contrairement aux présomptions, les travailleurs immigrés ne sont pas récemment arrivés : la moitié est au Canada depuis plus de dix ans et compte un minimum de cinq ans d'ancienneté dans l'entreprise. La majorité des entreprises participantes œuvre dans des secteurs où la surveillance de la SST est imposée (produits chimiques, matières plastiques, produits et transformation des métaux, bois et transports routiers). Les PE de l'échantillon emploient en moyenne trentehuit travailleurs, les trois quarts sont des hommes âgés de vingt-cinq à quarante-cinq ans, près de la moitié ont accompli douze années d'étude et quelques-uns ont une formation technique post-secondaire (15\%). Parmi un ensemble de variables comprises dans le modèle étudié des dynamiques de prise en charge des mesures de SST, nous retenons ici : a) la participation aux comités paritaires de SST au sein des entreprises, une pratique fortement encouragée par la loi ; b) les formations à l'embauche et en cours d'emploi dont les travailleurs ont bénéficié ; c) les attitudes manifestées par des arguments favorables et/ ou défavorables aux pratiques préventives de SST.

La seconde analyse décrit la participation des travailleurs immigrés et non immigrés à diverses activités de prévention à partir d'un questionnaire auto-administré $(n=171)$. Des vingt-huit entreprises de l'échantillon, dix-neuf ont participé à ce volet. Bien que le taux de participation des entreprises soit très satisfaisant, le taux de participation des travailleurs est de $30 \%$, soit 171 questionnaires complétés sur 610 . Les travailleurs ayant répondu à ce questionnaire sont majoritairement des hommes (69\%), âgés de quarantecinq ans et plus (61\%). Parmi ceux-ci, les immigrés sont plus scolarisés, $44 \%$ ayant un diplôme post-secondaire, contre 29 \% pour les travailleurs nés au Canada. En dépit des efforts pour assurer la participation des entreprises embauchant des travailleurs immigrés à ce deuxième volet, seulement $35 \%$ des travailleurs immigrés ont rempli ce questionnaire, contre $65 \%$ des travailleurs d'origine canadienne.

\section{Analyse comparative entre les entreprises embauchant majoritairement des immigrés et celles qui en embauchent peu ou pas}

La majorité des entreprises de notre échantillon (dix-neuf sur vingt-huit) ont un comité de SST. Toutefois, dans les entreprises embauchant une forte proportion d'immigrés, le comité est rarement paritaire. Il est le plus souvent composé de gestionnaires. Dans ces mêmes entreprises, le comité de SST est fonctionnel depuis peu de temps, deux ou trois ans tout au plus. La seule entreprise dotée d'un comité paritaire composé essentiellement de travailleurs immigrés (argentin, italien, haïtien) a connu une expérience unique : le comité s'est fait accompagner pendant plus de deux ans par une association sectorielle paritaire l'aidant à se constituer, à définir son mandat et à assigner des rôles à chacun de ses membres. De façon générale, les employeurs déplorent que les travailleurs immigrés ne veuillent pas participer à ce type de comité ni aux activités de prévention. Cependant, employeurs et employés issus de l'immigration peinent à comprendre le principe du paritarisme. 
Des dix-neuf entreprises embauchant des travailleurs immigrés, neuf n'offrent pas d'accompagnement structuré des nouveaux salariés. Les pratiques de compagnonnage ou de tutorat ne sont pas aussi fréquentes que dans les PE du groupe de comparaison, mais représentent un moyen de superviser les nouveaux employés (neuf sur dix-neuf vs six sur neuf). Le compagnon originaire du même pays ou maîtrisant les mêmes langues supervisera pendant quelques heures ou quelques jours le travail du nouveau collègue. Par exemple, dans une confiserie, la directrice de la production, cumulant la direction des ressources humaines et la coordination de la SST, d'origine canadienne, mais maîtrisant bien l'espagnol, constate un jour que ses nouveaux employés hispanophones (60\%) n'ont rien saisi des consignes de SST lors de leur initiation. Devant l'échec de cette formation dispensée par une firme externe, son entreprise décide de sélectionner un de ses employés hispanophones parmi les plus anciens pour développer ses compétences de formateur. On lui confie le mandat d'initier les nouveaux employés et d'assurer les mises à jour des formations en SST, et ce pour l'ensemble d'un personnel composé à $80 \%$ d'immigrés. Ainsi, certaines des entreprises développent leurs propres compétences pour former leurs travailleurs. Ce type d'initiative se retrouve surtout dans les entreprises mixtes où les dirigeants et les superviseurs sont d'origine canadienne tandis que les travailleurs sont immigrés. Aucune autre entreprise présentant une très grande homogénéité ethnique, autre que canadienne, n'a initié une telle démarche d'adaptation culturelle des formations pour ses employés.

Les responsables de la santé et sécurité au travail ont été interrogés sur les arguments favorables et défavorables à la SST entendus dans leur entreprise, qu'ils aient été tenus par des dirigeants, des superviseurs ou des travailleurs. Ce sont sensiblement les mêmes arguments qui ont été identifiés dans les deux types d'entreprises. Mais dans celles qui comptent le plus de travailleurs immigrés $(\geq 75 \%)$ plusieurs arguments défavorables ont été exprimés par les travailleurs et les superviseurs : " les mesures de SST demandent trop de temps, ralentissent la production, sont des contraintes et nuisent à l'exécution des tâches ». Malgré des dispositions législatives favorisant la participation des travailleurs aux comités de SST, à l'implantation de mesures préventives et curatives, aux enquêtes et aux autres mesures, les dynamiques de SST dans les petites entreprises ne démontrent pas une participation équitable entre travailleurs immigrés et non immigrés. Cette situation est confirmée par les travailleurs eux-mêmes.

\section{Analyse descriptive de la participation des immigrés}

Pour près de la moitié des interrogations du questionnaire auto-administré, les travailleurs immigrés ont donné des réponses négatives, statistiquement significatives $(\mathrm{p}<0,05)$, indiquant qu'ils ne bénéficient pas du même environnement en matière de santé et de sécurité au travail que les non immigrés (Tableau 1). 


\section{Tableau 1 : Participation et connaissance des travailleurs immigrés : des mesures de santé et de sécurité au travail}

\begin{tabular}{|c|c|c|c|c|}
\hline Question & Réponses & $\begin{array}{c}\text { Travailleurs } \\
\text { d'origine } \\
\text { canadienne }\end{array}$ & $\begin{array}{c}\text { Travailleurs } \\
\text { d'origine } \\
\text { étrangère }\end{array}$ & Total \\
\hline \multirow{2}{*}{ Comité de SST dans l'entreprise } & Oui & $91 \%(107)$ & $75 \%(47)$ & $85 \%(154)$ \\
\hline & Non & $9 \%(11)$ & $24 \%(16)$ & $15 \%(27)$ \\
\hline \multirow{2}{*}{ Formation à l'embauche } & Oui & $68 \%(80)$ & $47 \%(30)$ & $61 \%(110)$ \\
\hline & Non & $32 \%(38)$ & $52 \%(33)$ & $39 \%(71)$ \\
\hline \multirow{2}{*}{ Capacité à identifier des risques } & Oui & $91 \%(103)$ & $75 \%(44)$ & $81 \%(147)$ \\
\hline & Non & $9 \%(15)$ & $24 \%(19)$ & $19 \%(34)$ \\
\hline \multirow{2}{*}{ Entretien des machines } & Oui & $93 \%(110)$ & $84 \%(53)$ & $90 \%(163)$ \\
\hline & Non & $7 \%(8)$ & $16 \%(10)$ & $10 \%(18)$ \\
\hline \multirow{2}{*}{$\begin{array}{l}\text { Prévenir l'employeur lors d'une } \\
\text { blessure }\end{array}$} & Oui & $92 \%(109)$ & $76 \%(48)$ & $87 \%(157)$ \\
\hline & Non & $8 \%(9)$ & $24 \%(15)$ & $13 \%(24)$ \\
\hline \multirow{2}{*}{$\begin{array}{l}\text { Retour progressif, allègement de } \\
\text { tâche }\end{array}$} & Oui & $93 \%(107)$ & $84 \%(45)$ & $84 \%(152)$ \\
\hline & Non & $7 \%(11)$ & $16 \%(18)$ & $16 \%(29)$ \\
\hline \multirow{2}{*}{$\begin{array}{l}\text { Participer à l'enquête lors d'un } \\
\text { accident }\end{array}$} & Oui & $96 \%(115)$ & $89 \%(56)$ & $84 \%(152)$ \\
\hline & Non & $3 \%(3)$ & $11 \%(7)$ & $16 \%(11)$ \\
\hline \multirow{2}{*}{$\begin{array}{l}\text { Connaître les procédures en cas } \\
\text { d'accident }\end{array}$} & Oui & $81 \%(96)$ & $79 \%(44)$ & $77 \%(140)$ \\
\hline & Non & $19 \%(22)$ & $30 \%(19)$ & $23 \%(41)$ \\
\hline \multirow{2}{*}{$\begin{array}{l}\text { Aide pour compléter une demande } \\
\text { de réclamation }\end{array}$} & Oui & $81 \%(97)$ & $70 \%(43)$ & $77 \%(140)$ \\
\hline & Non & $19 \%(21)$ & $30 \%(20)$ & $23 \%(41)$ \\
\hline \multirow{2}{*}{ Procédures de sécurité à suivre } & Oui & $94 \%(111)$ & $84 \%(53)$ & $91 \%(164)$ \\
\hline & Non & $6 \%(7)$ & $19 \%(10)$ & $9 \%(17)$ \\
\hline \multirow{2}{*}{ Connaître les mesures d'urgence } & Oui & $95 \%(112)$ & $87 \%(55)$ & $92 \%(167)$ \\
\hline & Non & $5 \%(6)$ & $13 \%(8)$ & $8 \%(14)$ \\
\hline \multirow{2}{*}{$\begin{array}{l}\text { Équipement de protection } \\
\text { individuelle fourni par l'employeur }\end{array}$} & Oui & $87 \%(103)$ & $75 \%(47)$ & $83 \%(150)$ \\
\hline & Non & $13 \%(15)$ & $25 \%(16)$ & $17 \%(31)$ \\
\hline \multirow{2}{*}{$\begin{array}{l}\text { Port de l'équipement de protection } \\
\text { individuelle }\end{array}$} & Oui & $96 \%(113)$ & $84 \%(53)$ & $77 \%(140)$ \\
\hline & Non & $4 \%(5)$ & $16 \%(10)$ & $23 \%(41)$ \\
\hline
\end{tabular}

Les résultats montrent que les travailleurs immigrés reçoivent moins de formation à l'embauche que ceux d'origine canadienne (52\%vs $32 \%)$. De plus, ils sont moins compétents à identifier les risques (24\%vs 9\%), ils informent moins l'employeur lorsqu'ils se blessent ( $24 \%$ vs $8 \%$ ) et participent moins souvent aux enquêtes à la suite d'un accident (19\% vs $6 \%$ ). Plusieurs ne disposent pas d'équipement de protection $(25 \%$ vs $13 \%)$ et lorsque l'employeur en fournit un, ils sont moins enclins à le porter (16\%vs $4 \%)$.

Ces résultats confirment les observations faites par les responsables de la santé et de la sécurité au travail dans les entreprises : les travailleurs immigrés sont moins bien formés et informés en matière de SST, ils participent peu ou pas aux mesures préventives et correctrices, même lorsqu'il y a une enquête. Et finalement, le taux de participation des travailleurs immigrés à cette étude indique qu'ils ne se sentent même pas interpellés par une étude consacrée à leurs conditions. 


\section{LÉSIONS PROFESSIONNELLES CHEZ LES TRAVAILLEURS IMMIGRÉS, UNE AUTRE MANIFESTATION DES INÉGALITÉS SOCIALES DE SANTÉ}

Ces deux études, conduites indépendamment l'une de l'autre, indiquent des résultats convergents : les travailleurs immigrés victimes de lésions professionnelles connaissent des conditions défavorables, tant matériellement que socialement. Nos résultats apportent une vision fine des disparités qui sont, à l'image de nos sociétés, complexes, et ce, malgré un ensemble de structures tentant en vain de réduire les inégalités sociales de santé (Pampalon et al., 2008).

À l'instar du résultat des études de Kosny et al. (2011) et de Smith, Kosny et Mustard (2009), les résultats des deux études confirment que les travailleurs immigrés victimes de lésions professionnelles connaissent une situation matérielle inégalitaire qui se traduit par une précarité du revenu et du lien d'emploi. L'urgence à s'insérer sur le marché du travail mène les travailleurs à occuper des emplois où ils sont surexposés aux risques professionnels, tant parce que le secteur de production y est propice que parce qu'ils ne sont ni formés ni expérimentés à ces métiers de manœuvre. Ils sont embauchés dans des petites entreprises où les structures de SST sont minimalistes et parfois déficitaires (cas de la majorité), et où le contrat de travail est facilement rompu pour diverses raisons, dont le ralentissement de la production et la réduction du rendement des travailleurs blessés. Bref, une série de conditions entraînent des inégalités de revenu, qui constituent un second appauvrissement à la suite d'une lésion professionnelle.

Les travailleurs immigrés victimes de lésions professionnelles subissent également des inégalités sociales, mais qui ne sont pas nécessairement liées à un faible niveau d'éducation. En fait, dans les deux études, les travailleurs immigrés sont généralement un peu plus scolarisés que les travailleurs d'origine canadienne. Leur précarité sociale est davantage liée à la méconnaissance des droits d'exercer leur citoyenneté (Bibeau et Fortin, 2008). La méconnaissance des règles administratives du système d'indemnisation, des droits de recours en cas de refus ainsi que la maîtrise insuffisante de la langue pour décrire de façon explicite et sans ambiguités leurs souffrances, leurs lésions et les contextes dans lesquels elles sont apparues, limitent la capacité des travailleurs immigrés à exercer leurs droits.

À la suite des travaux de Kosny et al. (2011), la commission des lésions professionnelles de la province de l'Ontario a identifié des actions pour tenter de corriger les obstacles auxquels font face les immigrés dans l'exercice de leurs droits en matière de santé et de sécurité au travail. Une série de recommandations a émergé, invitant à la création de trousses d'information à l'intention des travailleurs immigrés, traduites en diverses langues et disponibles par Internet. Ces actions, bien qu'honorables, sont insuffisantes pour assurer une juste participation des immigrés aux mesures de SST dans leur entreprise. Car, outre leur manque d'information, d'autres éléments contribuent au faible engagement des immigrés, à commencer par le fait qu'ils aspirent très souvent à quitter rapidement ces emplois pour lesquels ils sont surqualifiés. Parallèlement, les employeurs voient mal l'intérêt d'impliquer ces travailleurs qui cherchent à être embauchés ailleurs. Alors que 
les résultats indiquent, contre toute attente, que les travailleurs immigrés occupent ces emplois plus de cinq ans, voire plus de dix ans, les représentations des uns et des autres découragent l'implication favorable à la santé et la sécurité au travail. Cette stabilité inattendue s'explique, selon les responsables de la SST des petites entreprises interrogés dans le cadre de la seconde étude, par le rôle du collectif de travail. Entourés de collègues également issus de l'immigration et très souvent originaires de la même communauté, ces travailleurs maintiennent, au-delà de leur aspiration, leur lien d'emploi avec l'entreprise qui a servi de tremplin à leur insertion professionnelle. Comment expliquer alors que les entreprises qui embauchent des travailleurs immigrés investissent peu ou pas dans les mesures de SST et ne favorisent pas la contribution de ces travailleurs qui, malgré tout, leur sont loyaux?

Outre les explications structurelles et financières, les résultats de notre étude sur les dynamiques de SST dans les petites entreprises montrent que ces dynamiques sont conditionnées à la fois par la formation des dirigeants et par leurs origines. Les dirigeants, les propriétaires et les cadres, qui ont suivi une formation au Canada en gestion, en ressources humaines ou en production, connaissent mieux les lois de SST et donc, les droits et obligations des travailleurs et des employeurs. Forts de leurs connaissances, ces dirigeants déploient des efforts afin d'impliquer tous les travailleurs sans exception aux mesures de santé et de sécurité au travail. Ce faisant, ils visent également à retenir une main-d'œuvre de plus en plus difficile à renouveler. Conscients de la complexité de la loi de SST, complexité souvent exacerbée par les écarts interculturels de compréhension des droits et des obligations, ces gestionnaires formés au Canada sont plus soucieux d'adapter les pratiques de santé et de sécurité aux travailleurs issus de diverses communautés. Ils sont plus attentifs aux besoins d'adaptation culturelle des mesures de SST que leurs homologues immigrés, diplômés à l'étranger (Gravel, Rhéaume et Legendre, 2011).

\section{CONCLUSION}

Comme Bibeau et Fortin (2008), nous déplorons la tendance réductionniste des recherches en santé publique qui étudient les inégalités sociales de santé par la mesure des associations entre les taux de morbidité, de mortalité ou de mortalité prématurée et les niveaux d'éducation et de revenus. La pauvreté et la vulnérabilité prennent diverses facettes et sont trop souvent sous-estimées dans les études en milieu urbain. C'est le cas chez les travailleurs immigrés qui, malgré un taux d'éducation plus élevé et un revenu salarial, connaissent des situations d'inégalités sociales de santé parce qu'ils occupent des emplois pour lesquels ils n'ont ni les compétences, ni les savoirs faire requis, des emplois d'insertion sur le marché du travail pour lesquels ils sont souvent surqualifiés. Inégalités de santé qui se doublent de discriminations - différences de traitement injustes et évitables - en matière d'indemnisation ou de retour au travail en cas de lésions professionnelles.

Il faut être en mesure de documenter les conditions qui pénalisent des travailleurs pauvres peinant à conserver leur emploi. Trop souvent exclus des modèles prévisionnels des inégalités sociales de santé parce qu'ils ont un revenu à peine supérieur au seuil de pauvreté, les travailleurs immigrés sont également exclus des analyses des inégalités maté-

REMI 2012 (28) 2 pp. 57-80 
rielles et sociales parce qu'ils sont trop scolarisés et surqualifiés pour les emplois qu'ils occupent. Pourtant, ces travailleurs immigrés, doublement victimes de lésions professionnelles qui les entraînent dans une spirale d'inégalités sociales et d'inégalités de santé ${ }^{8}$, sont des acteurs importants pour l'essor économique des sociétés industrialisées en situation de pénurie de main-d'œuvre.

\section{Références bibliographiques}

ALCORSO Caroline (1988) Migrant Workers and Workers' Compensation in New South Wales, Kensington, Social Welfare Research Centre, 168 p.

AMODU Tola (2008) The determinants of compliance with laws and regulations with special reference to health and safety. A literature review, London School of Economics and Political Science for the Health and safety executive (HSE), Report RR-638, 58 p.

AZAROFF Lenore S., LEVENSTEIN Charles and WEGMAN David H. (2002) Occupational Injury et Illness Surveillance: Conceptual Filters Explain Underreporting, American Journal of Public Health (AJPH), 92 (9), pp. 1421-1429.

BENZÉCRI Jean-Paul (1994) Correspondence Analysis Handbook, New York, Marcel Dekker, $665 \mathrm{p}$.

BERTHELETTE Diane et PLANCHÉ Frédéric (1995) Évaluation des programmes de sécurité du travail dans des PME. Études et recherches IRSST (Rapport R-107), Montréal, 56 p.

BIBEAU Gilles et FORTIN Sylvie (2008) Inégalité et souffrance sociale : une approche anthropologique, in Katherine Frolich, Maria De Koninck, Andrée Demers et Bernard Paul, Les inégalités sociales de santé au Québec, Montréal, Presses Université de Montréal, pp. 111-139.

BOUCHARD Pierre (2008) Les entreprises de 11 à 49 employés : portrait de leur réalité linguistique. Office québécoise de la langue française, Gouvernement du Québec, 56 p.

BROWN Mariane P., DOMENZAIN Alejandra and VILLORIA-SIEGERT Nelliana (2002) California's Immigrant Workers Speak Up About Health and Safety in the Workplace, Health \& Safety policy brief, Labor occupational safety and health program (LOSH), $4 \mathrm{p}$.

DEMBE Allard E., SUM Juliann, BAKER Christine, STOMBERG Kristen and NEMIROVSKY Irina (2003) Workers' Compensation Medical Care in California: Costs, Oakland, California, California Healthcare Foundation and the California Commission on Health and Safety and Workers' Compensation, pp. 1-4.

DESMARAIS Lise (2004) Évaluation de l'implantation des comités de santé et de sécurité du travail : une étude de cas multiples réalisée dans les petites et moyennes entreprises au Québec. Thèse de doctorat, Montréal, Université du Québec à Montréal, 319 p.

EAKIN Joan, LAMM Felicity and LIMBORG Hans Jorgen (2000) International perspective on the promotion of health and safety in small workplace, in Kaj Frick, Per Langaa Jensen, Michael Quinlan and Ton Wilthagen, Systematic occupational health and safety management: perspectives on an international development, Oxford, Pergamon Press, pp. 227-247.

FRUMKIN Howard, WALKER E.-D. and PRANSKY Glenn (1999) Minority Workers and Communities. Occupational Medicine, Special Populations, 14 (3), pp. 495-517.

GODIN Jean-François, PINSONNEAULT Gérard, BENZAKOUR Chakib, TRUONG Minh, AUDET Benoît et MAHER France (2004) L'insertion en emploi des travailleurs admis au Québec en vertu de la grille de sélection 1996, Direction de la population et de la recherche, MRCI, Gouvernement du Québec. 74 p.

8 Voir aussi sur ce point l'article de Cognet et al. dans ce numéro. 
GRAVEL Sylvie (2006) Analyse du parcours d'indemnisation de travailleurs immigrants victimes de lésions professionnelles. Thèse de doctorat, Facultés des études supérieures de l'Université de Montréal, pour l'obtention d'un Ph.D. en santé publique. 296 p.

GRAVEL Sylvie, BOUCHERON Laurence et KANE Michel (2003) La santé et la sécurité au travail des travailleurs immigrants à Montréal : résultats d'une enquête exploratoire, Perspectives Interdisciplinaires Sur le Travail Et la Santé (PISTES), 5 (1), 12 p.

GRAVEL Sylvie, BRODEUR Jean-Marc, CHAMPAGNE François, LIPPEL Katherine, PATRY Louis, BOUCHERON Laurence, FOURNIER Michel et VISSANDJÉE Bilkis (2006) Critères pour apprécier les difficultés d'accès à l'indemnisation des travailleurs victimes de lésions professionnelles, Perspectives Interdisciplinaires Sur le Travail Et la Santé (PISTES), 8 (2), $15 \mathrm{p}$.

GRAVEL Sylvie, RHÉAUME Jacques et CYR Hélène (2008) Les stratégies favorables au développement et au maintien de mesures de santé et de sécurité au travail dans les petites entreprises du centre urbain montréalais embauchant une main-d'œuvre immigrante. Protocole accepté IRSST, Printemps 2008, Dossier n 099-682, 49 p.

GRAVEL Sylvie, RHÉAUME Jacques and LEGENDRE Gabrielle (2011) Strategies to develop and maintain occupational health and safety measures in small businesses employing immigrant workers in metropolitan Montreal, International Journal of Workplace Health and Management (IJWHM), 4 (2), pp. 164-178.

GRAVEL Sylvie, VISSANDJÉE Bilkis, LIPPEL Katherine, BRODEUR Jena-Marc, PATRY Louis and CHAMPAGNE François (2010) Ethics and Compensation of Immigrant Workers for WorkRelated Injuries and Illnesses, Journal of Immigration and Minority Health, 12 (5), pp. 707-714.

HEALTH AND SAFETY EXECUTIVE (HSE) (2005) Occupational health and safety support systems for small and medium sized enterprises. A Literature Review, Bomel Limited for the Health and safety executive (HSE) 2005, Report RR-410, 129 p.

HENSHAW John (2002) United States Seatle Subcommittee on Employment Safety and Training, Washington DC, Occupational Health and Safety Administration (OSHA).

HERBERT Robin, JANEWAY K. and SCHECHTER C. (1999) Carpal Tunnel Syndrome and Workers' Compensation among an Occupational Clinic Population in New York State, American Journal of Industrial Medicine, 35 (4), pp. 335-342.

HIMMELSTEIN Jay S. and PRANSKY Glenn S. (2003) Measuring and Improving Quality of Workers' Compensation Medical Care, in Allard E. Dembe, Sharon E. Fox et Jay S. Himmelstein, Improving Quality of Workers' Compensation Medical Care: A National Challenge, Beverly MA, OEP Press, chap. 7.

KOSNY Agnieszka, LIFSHEN Marni, MACEACHEN Ellen, SMITH Peter, JAFRI Gul Joya, NEILSON Cynthia, PUGLIESE Diana and SHIELD John (2011) Delicate Dances: immigrant workers' experiences of injury reporting and claim filing, Institute for Work and Health, Study report, $38 \mathrm{p}$.

LANSDOWN Terry C., DEIGHAN Caroline and BROTHERTON Chris (2007) Health and safety in the small to medium-sized enterprise. Psychosocial opportunities for intervention, Heriot Watt University for the Health and Safety Executive (HSE), Report RR-578, 100 p.

LASHUAY Nan, BURGEL Barbara J., HARRISON Robert, ISRAEL Leslie, CHAN Jacqueline, CUSIC Catherine, CHAO PUN Jane, FONG Ken and SHIN Young (2002) We spend Our Days Working in Pain: A Report on Workplace Injuries in the Garment Industry, Asian Immigrant Women Worker Clinic, Oakland, California, 26 p.

LEE Tau Lee, KRAUSE Niklas, GOETCHIUS Charles, ARGRIESTI Jo Marie and BAKER Robin (2003) Participatory action research with hotel room cleaners: from collaboration study to the bargaining table in Meredith Minkler et Nina Eallerstein, Community Based Participatory Research for Health. Form process to outcomes, San Francisco, California, Jossey-Bass, chap. 19. 
MENDELOFF John, NELSON Christopher, KO Kilkon and HAVILAND Amelia (2006) Small Businesses and Workplace Fatality Risk - An Exploratory Analysis, Kauffman-RAND Center for the Study of Small Business and Regulation (TR-371), $104 \mathrm{p}$.

MURRAY Linda Rae (2003) Sick and Tired of Being Sick and Tired: Scientific Evidence, Methods and Research Implications for Tacial and Ethnic Disparities in Occupational Health, American Journal of Public Health, 93 (2), pp. 221-226.

PAMPALON Robert et RAYMOND Guy (2003) Indice de défavorisation matérielle et sociale : son application au secteur de la santé et du bien-être. Santé, Société et Solidarité 2003, 2 (1), pp. 191-208.

PAMPALON Robert, HAMEL Denis et GAMACHE Philippe (2008) Évolution de la mortalité prématurée au Québec selon la défavorisation matérielle et sociale, in Katherine Frolich, Maria de Koninck, Andrée Demers et Bernard Paul, Les inégalités sociales de santé au Québec, Presses Université de Montréal, pp. 13-35.

PATRY Louis, GRAVEL Sylvie, BOUCHERON Laurence, FOURNIER Michel, KANE Michel, BEAUVAIS Julie et VISSANDJÉE Bilikis (2005) Accès à l'indemnisation des travailleurs et travailleuses immigrant(e)s victimes de lésions musculo-squelettiques d'origine professionnelle. Rapport de recherche, Direction de la santé publique de Montréal, Fonds québécois de recherche sur la société et la culture (FQRSC) n 94577 SR-4658.

PEEK-ASA Corinne, ERICKSON R. and KRAUSS Jess F. (1999) Traumatic Occupational Fatalities in the Rentail Industry, United States 1992-1996, American Journal of Industrial Medicine, 35 (2), pp. 186-191.

PRANSKY Glenn S., BENJAMIN Katy, HILL FOTOUHI Carolyn, HIMMELSTEIN Jay S., FLETCHER Kenneth E., KATZ Jeffrey N. and JOHNSON William G. (2000) Outcomes in Work-Related Upper Extremity and Low Back Injuries: results of retrospective Study, American Journal of Industrial Medicine, 37, pp. 400-409.

QUINLAN Michel and MAYHEW Claire (2000) Precarious employment, work re-organisation and the fracturing of OHS management, in Kaj Frick, Per Langaa Jensen, Michael Quinlan and Ton Wilthagen, Systematic occupational health and safety management: perspectives on an international development, Oxford, Pergamon Press, pp. 175-198.

ROSENMAN Ken D., GARDINER Joseph C., WANG J., BIDDLE Jeff E., HOGAN A., REILLY M.-J., ROBERTS K. and WELCH Ed (2000) Why Most Workers with Occupational Repetitive Trauma do not file for Workers' Compensation, Journal of Occupational and Environmental Medicine, 42 (1), pp. 25-43.

RoSPA (1992) Minority report, Occupational safety and Health, November.

RUDOLPH Linda, DERVIN Kathy, CHEADLE Allen, MAISLISH Neil and WICKIZER Tom (2002) What Do Injured Workers Think About Their Medical Care and Outcomes after Work Injury?, Journal of Occupational and Environmental Medicine, 44 (5), pp. 425-434.

SCHERZER Teresa, RUGULIES Reiner and KRAUSE Niklas (2005) Work-Related Pain and Injury and Barriers to Workers' Compensation among Las Vegas Hotel Room Cleaners, American Journal of Public Health, 95 (3), pp. 483-488.

SEN Amartya (2009) Quelle égalité ?, in Amartya Sen, Éthique et économie, Autres essais $n^{\circ}$ IV, Quadrige, Paris, PUF, pp. 189-213.

SMITH Peter, KOSNY Agnieszka and MUSTARD Cameron (2009) Differences in access to wage replacement benefits for absences due to work-related injury or illness in Canada, American Journal of Industrial Medicine, 52 (4), pp. 341-349.

SMITH Peter and MUSTARD Cameron (2009) Comparing the risk of work-related injuries between immigrants to Canada, and Canadian-born labour market participants, Occupational and Environmental Medicine, 66 (6), pp. 361-367.

SMITH Peter and MUSTARD Cameron (2010) The unequal distribution of occupational health and safety risks among immigrants to Canada compared to Canadian-born labour market participants: 1993-2005, Safety Science, 48 (10), pp. 1296-1303. 
STATISTIC SWEDEN (2001) Invandrarnas levnadsförhallanden'i Välfärd och ojämlikhet I 20-arsperspekytiv 19975-19995, Rapport 91, in Karen Wren and Paul Boyle, Migration and work-related health in Europe; A literature Review, National Institute for Working Life and SALTSA. Migration, work environment and Health, documentation from workshop, SALTSA Report 2000, 1, Stockholm, Sweden.

STATISTIQUE CANADA (2010) Établissement employeurs selon la taille de l'établissement dans les provinces et territoires, décembre 2009. Registre des entreprises, Internet.

STRUNIN Lee and BODEN Leslie I. (2004) The Workers' Compensation System: Worker Friend or Foe?, American Journal of Industrial Medicine, 45 (4), pp. 338-345.

SUM Juliann (1996) Navigating the California Workers' Compensation System: The injured Worker's Experience. An Evaluation of Services to Inform and Assist Injured Workers in California, University of California at Berkeley (San Francisco) for the Commission on Health and Safety and Workers' Compensation, [en ligne] consulté le 10 décembre 2009. URL : http:// www.lohp.org/docs/pubs/navigate.html.

TERAN Suzanne, BAKER Robin and SUM Juliann (2002) Improving Health and Safety Conditions for California's Immigrant Workers, WISH coalition, California Working Immigrant Safety and Health, $44 \mathrm{p}$.

UNDERHILL Elsa and QUINLAN Michael (2011) How Precarious Employment Affects Health and Safety at Work: The Case of Temporary Agency Workers, Relations industrielles, 66 (3), pp. 397-421.

VINCENT François et GAUDREAULT Simon (2011) L'intégration par l'emploi, le développement du Québec par l'immigration: une solution pour les PME, Fédération canadienne de l'entreprise indépendante. Consultation sur la planification de la main-d'œuvre, pp. 1-16, [en ligne]. URL : http://www.cfib-fcei.ca/cfib-documents/qc1028.pdf

WCB UPDATE (2008) Developing Safety Resources for Immigrant Workers: The Manitoba Immigrants' Safety Initiative (MISI).

WINDAU Janice (1997) Occupational Fatalities among the Immigrant Population, Compensation and Working Conditions, pp. 40-44. 


\title{
Les inégalités sociales de santé des travailleurs immigrés au Québec victimes de lésions professionnelles
}

\author{
Sylvie Gravel, Jacques Rhéaume et Gabrielle Legendre
}

\begin{abstract}
Deux études sur la santé et sécurité au travail (SST) des immigrés sont retenues pour discuter des inégalités sociales de santé. La première a documenté les parcours d'indemnisation des travailleurs immigrés victimes de lésions professionnelles $(n=104)$. Les résultats indiquent que ces travailleurs doivent surmonter plus d'obstacles que les Canadiens lors des consultations médicales et juridiques, des procédures administratives et lors de la réintégration au travail, un parcours menant souvent au licenciement. La seconde étude était centrée sur l'implantation des mesures de SST dans les petites entreprises ( $<50$ employés) embauchant des immigrés ( $>25 \%)$. Dans ces entreprises $(n=28)$, les travailleurs immigrés sont moins bien formés à la SST, identifient mal les risques, déclarent peu leurs lésions et participent rarement aux enquêtes d'accident. Certains ne portent pas d'équipement de protection. Les inégalités sociales de santé se manifestent ici par les limitations fonctionnelles du travailleur, son licenciement à la suite d'une lésion et son incapacité à exercer un recours.
\end{abstract}

\section{Social Inequalities in Health of Immigrant Workers in Quebec Victims of Occupational Injuries}

\author{
Sylvie Gravel, Jacques Rhéaume and Gabrielle Legendre
}

Two studies on migrants' health and safety measures (OHS) at work are selected to discuss social inequalities in health. The first study documented the trajectories of immigrant workers' compensation victims of occupational injuries $(n=104)$. The results indicate that immigrant workers face more obstacles than Canadian workers during medical and legal consultations, during administrative procedures and during the reinstatement in the work. The trajectories often lead to dismissal. The second study focused on the implementation of OSH measures in small businesses $(<50$ employees) hiring immigrants $(>25 \%)$. In these businesses $(n=28)$, immigrant workers are less well trained in OSH, misjudge risks, few declare their injuries, and rarely participate in accident investigation. Many do not wear protective equipment. Immigrant workers who are victims of occupational injuries live with permanent disabilities that impair their productivity and often lead to a dismissal, for which they don't know their appeals. This is a pathway of social inequalities in health. 


\title{
Las desigualdades sociales a la salud de los trabajadores emigrantes en Quebec víctimas de lesiones profesionales
}

\author{
Sylvie Gravel, Jacques Rhéaume y Gabrielle Legendre
}

Dos estudios sobre la salud y la seguridad en el trabajo (SST) de los emigrantes son retenidos para discutir las desigualdades sociales a la salud. La primera documentó los trayectos de indemnización de los trabajadores inmigrantes víctimas de lesiones profesionales $(n=104)$. Los resultados indican que estos trabajadores deben superar más obstáculos que los canadienses en el momento de las consultas médicas y jurídicas, los procedimientos administrativos y en el momento de la reintegración en el trabajo. Un trayecto que a menudo lleva al despido. El segundo estudio fue centrado sobre la implantación de las medidas de SST en las pequeñas empresas $(<50$ empleados $)$ que contrataban a inmigrantes $(>25 \%)$. En estas empresas $(n=28)$, los trabajadores inmigrantes son formados menos bien en el SST, identifican mal los riesgos, declaran poco sus lesiones, y participan raramente en las encuestas de accidente. Varios no llevan equipo de protección. Las desigualdades sociales a la salud se manifiestan aquí por las limitaciones funcionales del trabajador, su despido en consecuencia de una lesión, y sus incapacidades que ejercen un recurso. 